\title{
Mindfulness-Based Interventions for Successful Aging: Benefits, Barriers, and the Future
}

\author{
Jamie M. Sulek ${ }^{1}$ \\ sulekj@uvic.ca
}

\begin{abstract}
A growing body of research supports the efficacy of mindfulness-based interventions (MBIs) to address a wide range of health concerns. This narrative literature review recognizes the important role these interventions play in response to public health challenges prompted by an aging demographic. Moreover, this review identifies opportunities to deliver cost-effective, communitybased interventions tailored to older adults that promote successful aging. Complementary to this diverse demographic, MBIs allow participants to access naturally developed abilities that support overall well-being and resiliency later in life. The intersecting and beneficial effects of these interventions offer a well-suited, proactive, and holistic avenue to enhance quality of life for older adults.
\end{abstract}

Keywords: mindfulness; intervention; successful aging; older adults; well-being

\footnotetext{
${ }^{1}$ I would like to acknowledge with gratitude both Dr. Lori Harper, Athabasca University and Dr. Emily Arvay, University of Victoria, for their guidance, feedback, and encouragement.
} 


\section{Mindfulness-Based Interventions for Successful Aging: Benefits, Barriers, and the Future}

Western societies currently face significant public health challenges prompted by the demands associated with increased life expectancy and an aging demographic (Lomas et al., 2016). Specifically, older adults represent the fastest-growing cohort with the highest rates of health care needs (Hazlett-Stevens et al., 2019). This rising demand of seniors seeking medical care to address chronic medical conditions necessitates the urgent need for cost-effective ways to promote the health and well-being of an aging population (Hazlett-Stevens et al., 2019; Lomas et al., 2016).

A growing body of research supports the efficacy of mindfulness-based interventions (MBIs) for a "variety of populations to treat a wide range of physical and psychological disorders" (Kocovski et al., 2009, p. 85). Although a priority for the field, research on the processes through which mindfulness affects well-being remains in its infancy, especially research that targets older adults (Geiger et al., 2016). With the efficacy of MBIs supported across multiple treatment domains, there is considerable room for the creation of MBIs designed to address the needs of older adults that encourage "successful aging" rather than the treatment of "disfunction [or] disorder" (Lomas et al., 2016).

Importantly, a study by Hazlett-Stevens et al. (2019) reminds us that the primary aim of MBIs is the "intentional practice of acceptance" rather than the targeting of "symptoms for change" (p. 349). Moreover, with a variety of stressors that "hinder [the] health-related quality of life" for older adults, protective factors that buffer against these stressors fostered through MBIs provide a "mechanism for effective self-regulation and stress reduction" (de Frias \& Whyne, 2015, p. 201). With expanding MBI research that supports a vast array of health-related benefits, how might future interventions be tailored and applied proactively to better serve older adults and society at large? This narrative literature review explores research using a successful aging perspective to better understand the application of MBIs for older adults, which may guide the future delivery of these interventions to this cohort - a topic of interest to scholars, facilitators, and clinicians who work in this domain.

In selecting literature for this review, inclusion criteria included studies from the last 10 years that explored the delivery of MBIs to older adults (over the age of 65). Although inclusion criteria for two of the three studies showcased restricted participants using a chronological cut-off to address an identified gap in MBI research for this cohort specifically, it is essential to acknowledge the diversity within this demographic, which cannot be defined by chronological age. This point is further expanded upon in the limitations section of this review. The University of Victoria libraries' search engine was used to identify the articles included in this study, and key search terms included mindfulness, aging, and older adults. More specifically, of the three studies featured in this review, one provides a unique micro-level perspective (real-time focus group discussion), another provides a macro-level perspective (comprehensive review of 15 studies), and the third provides a lifespan perspective highlighting key developmental theories. Together, this research offers both a well-rounded and introductory look at the delivery of MBIs to this growing demographic that can enlighten future work in this increasingly important field.

First, to provide both the context for and scope of this review, the concepts of mindfulness, MBIs, and successful aging will be defined, followed by a summary of recent literature highlighting the natural alignment of MBIs with developmental theories. Next, this discussion is

followed by exploratory research that offers the benefits, barriers, as well as recommended modifications for the effective delivery of MBIs to older adults. Lastly, this review closes by 
discussing research limitations and possibilities for future research that center on the delivery of MBIs to promote successful aging.

\section{Definitions: Mindfulness, MBIs, and Successful Aging}

Recent literature debates the definition and optimal measurement of mindfulness as a multifaceted construct (Grossman, 2019). Although the term mindfulness has been used to mean many things, this review cites Kabat-Zinn's work, which describes mindfulness as "the awareness that emerges through paying attention on purpose, in the present moment, and non-judgmentally to the unfolding of experience moment by moment" (2003, p. 145). Further, it should be highlighted that the engagement in mindfulness practice is not limited to a particular set of techniques; it includes formal (i.e., structured practice) and informal practices that aim to "cultivat[e] a continuity of awareness in all activities of daily living" (Kabat-Zinn, 2003, p. 147). Although a multifaceted construct, mindfulness can be described as the invitation and acceptance of all that arises in the present moment, regardless of time and setting.

Among the many forms of intervention, this review focuses on two of the most widely studied MBIs that have gained empirical support, which include Mindfulness-Based Stress Reduction (MBSR) and Mindfulness-Based Cognitive Therapy (MBCT), as well as modifications to these approaches required to meet the needs of older adults (Berk et al., 2017; Hempel et al., 2014). MBSR was developed by Kabat-Zinn in 1979 and is based on Buddhist traditional meditation practices (Kabat-Zinn, 2003). Originally, the practice was developed to treat chronic pain and is now applied to a much broader spectrum of health conditions (Hazlett-Stevens et al., 2019; Kabat-Zinn, 2003). Traditional MBSR and MBCT share 8-week group formats as well as mindfulness meditation components, which include sitting meditation, body scans, and hatha yoga (Berk et al., 2017; Kabat-Zinn, 2003). MBCT adds to traditional MBSR with its cognitive therapy elements and disorder-specific psycho-education (Xie et al., 2014).

Like the concept of mindfulness, the term "successful aging" is multifaceted and continues to evolve; however, this review defines successful aging as "high physical, psychological and social functioning in old age without major diseases" (Rowe \& Kahn, 1997, p. 433). Specifically, an evolving body of research addresses the significant influence of individual lifestyle factors that contribute to a range of aging characteristics in addition to other important factors such as genetics. This extended view of successful aging suggests that although the risk of disease and disability can be age-related, it is not age-dependent. Further, the concept of successful aging is proactive and encourages the maintenance of high physical and cognitive functions, sustained engagement, as well as avoidance of disease and disability.

\section{Developmental Theory}

The use of MBIs for older adults is well aligned with existing developmental theory, and this section introduces some key theories that suggest that the natural process of aging often results in increased mindfulness. A recent study by Mahlo and Windsor (2020) references lifespan developmental theories concerned with aging and shows that aspects of mindfulness, such as "present-moment attention, nonjudgement, [and] acceptance," complement the motivational preferences of older adults and promote resilience in response to age-related challenges (p. 1).

First, Carstensen's Socioemotional Selectivity Theory (SST) suggests that, as individuals age and become increasingly aware of their own mortality, they become more present-minded with 
heightened motivation to foster more meaningful lives (Carstensen, 2006; Mahlo \& Windsor 2020). Interestingly, SST demonstrates that, young or old, when individuals perceive time as finite, their priorities shift from gathering information and expanding their horizons to finding emotional meaning and satisfaction in life (Carstensen, 2006).

Next, the Dual Process Model (DPM) developed by Brandtstadter (2009) suggests that as individuals age, there is an erosion of the belief that they are in control, which is accompanied by the understanding that "any person's biography is a mixture of desired and undesired, intended and unintended changes or events" (pp. 52-54). The DPM postulates that, as individuals age and experience irreversible losses, working through those losses sets the stage for more accommodative and adaptive ways of being. This process in turn allows individuals to let go of unattainable ambitions, acknowledge their current circumstances, and reorient toward new goals (Brandtstadter, 2009; Farb et al., 2014 as cited in Mahlo \& Windsor 2020).

Lastly, the Strength and Vulnerability Integration (SAVI) model suggests that, in connection with SST, aging is accompanied by the motivation to limit one's exposure to negative experiences (Charles, 2010; Mahlo \& Windsor, 2020). This model acknowledges both age-related strengths (e.g., attentional strategies, appraisals, and emotional regulation), but also points to agerelated vulnerabilities that negatively influence one's recovery from sustained and high-stress situations (Charles, 2010). As a result, one's increased vulnerability to unavoidable stressors late in life make mindfulness traits both protective and restorative in nature (Charles, 2020; Mahlo \& Windsor, 2020).

In brief, coming to terms with mortality results in people creating more meaningful lives that work within their current circumstances and limit their exposure to negative experiences. Taken together, these theories suggest that normative developmental changes (i.e., becoming more present-minded and accommodative) support one's well-being and resiliency later in life.

\section{Research: MBIs for Older Adults}

With a growing body of research that endorses the many pathways through which MBIs can influence well-being, programs are now offered in hospitals, clinics, schools, workplaces, prisons, and community health settings to share a vast range of benefits (Crane et al., 2017). To establish both the efficacy and effectiveness of MBIs, research to date has been conducted primarily with young or middle-aged adults; however, given promising results, there is an increased need to focus research on older adults who represent the fastest growing segment of the population (Geiger et al., 2016; Hazlett-Stevens et al., 2019). Given the potential of these interventions, research continues to expand and support the value of MBIs for older adults who seek to strengthen their awareness and attention capacities, improve cognitive functioning, manage chronic pain and illness, as well as reduce anxiety, depression, and other forms of psychological distress (Hazlett-Stevens et al., 2019). Despite a broad range of treatment benefits, this review highlights the value of MBIs from a successful aging perspective with a focus on enhancing wellbeing rather than symptom reduction. The following studies have been selected to showcase the various ways in which MBIs are studied and explored with older adults, including both macroand micro-level perspectives intended to inform future research.

Incorporating the developmental theories outlined above, the study by Mahlo and Windsor (2020) on dispositional mindfulness in older adults found that attributes, such as present-moment attention, acceptance, and nonjudgement, were positively associated with age. Through time and experience, these naturally developed abilities facilitated mindfulness-related capacities that 
allowed participants to be flexible and adaptable when faced with changing circumstances, as well as to positively influence their overall perception of well-being with age.

In a small 2016 pilot study, a brief "positive aging" MBI called Mindful Living in Older Age (MLOA) modified traditional MBSR to address the needs of healthy older adults aged 65 to 75 (Lomas et al., 2016, p. 631). The focus of this pilot study was to create a MBI tailored to older adults that could be delivered in the community rather than in a traditional clinical setting to promote successful aging. Results were collected from six participants using a qualitative survey and recorded in real-time focus group discussions throughout the study to capture the benefits, barriers, as well as detailed participant insights to inform future MBIs for older adults. While many valuable barriers were identified, this study demonstrated that participants found MLOA useful, effective, and successful in introducing the practice as well as encouraging its ongoing use in a cost-effective manner. This small pilot study offered rich qualitative insights and guidance for future clinicians and researchers to deliver MBIs to older adults.

In a 2016 review of 15 studies using traditional MBSR, MBCT, or modified forms to facilitate the needs of older participants, the effects on physical and emotional well-being were measured primarily using self-reported instruments (Geiger et al., 2016). The findings of this study were exploratory in nature given the limited research available and provided further support that MBIs for older adults are both acceptable and feasible. The limited size of this study and the inconsistency among incorporated research (i.e., lack of consensus on modifications) was determined to be the most important finding in Geiger et al.'s review. This study highlights the need for more rigorous research, representative population samples, and consensus on intervention modifications, which together can inform a formalized treatment for this older demographic. Sample sizes with a mean age of 65 to 83 ranged from 20 to 228 participants with the majority being female and Caucasian - a consistent limitation across the many studies included in this review.

\section{The Benefits}

The World Health Organization defines health as "a state of complete physical, mental and social well-being and not merely the absence of disease or infirmity," and this multifaceted definition speaks to the equally multifaceted burdens of old age (World Health Organization, 2021; Lomas et al., 2016, p. 631). Following suit, a comprehensive approach to health care that is not only holistic but also cost-effective, proactive, and non-pharmacological, make interventions like MBIs well suited to older adults with increasing needs (Hazlett-Stevens et al., 2020). Further, it is important to highlight that multidimensional approaches to health and well-being have value at both individual and societal levels, even if only considered from an economic perspective (Lomas et al., 2016).

A recent qualitative review that looked at MBIs (MBSR and MBCT) for older adults who experienced a range of physical, behavioral, and cognitive challenges highlighted that the mere practice of acceptance without motive resulted in symptom improvement (Hazlett-Stevens et al., 2020). Paradoxically, although MBSR emphasizes the principle of "acceptance [vs.] change," the more participants practiced in the moment acceptance of discomfort, the more they noticed symptom improvements (Kabat-Zinn, 2013 as cited in Hazlett-Stevens et al., 2020, p. 355). In other words, when participants focused on accepting their reality, in the moment, without resistance or the motive of change, participants often indirectly experienced reduced symptoms. 
When approached as an "asset-based" intervention, one that proactively enhances wellbeing rather than targets dysfunction, there is an opportunity to improve the quality of life for those who are currently well but at risk of developing physical and mental health issues in the future (Lomas et al., 2016, p. 631). MBIs designed and delivered in the community that promote successful aging also work to stall the onset of future health issues, an outcome beneficial at both individual and societal levels. Moreover, an important finding from the study on dispositional traits suggested that the development of mindfulness-related capacities in older age, regardless of how they are acquired, give rise to an ability to be flexible and adaptable to life's circumstances while positively influencing overall perceptions of well-being (Mahlo \& Windsor, 2020). Further, because MBIs emphasize a willingness to accept and experience the present moment, when applied to an older demographic, mindfulness practice allows participants to tap into and capitalize on inherent strengths - such as exercising the natural emotional regulation strategies that develop with age (Geiger et al., 2016; Wingate et al., 2005).

A small pilot study that delivered a modified brief MBI (MLOA) reported many positive themes among participants after completion, including a new and enhanced sense of calm, exploration, and savoring of simple daily activities (Lomas et al., 2016). Participants also noted a newfound realization that they had acquired a useful tool for coping with stress and discomfort in their lives. These findings echo findings from more extensive clinical programs, which suggest that modified brief programs like MLOA delivered in the community could allow participants to access the same benefits achieved in more rigorous and lengthy clinical programs. To this point, a study that compared the outcomes of a standard 12-week MBSR program with an 8-week program found the longer program to be no more effective (Lenze et al., 2014. This conclusion was contrary to the hypothesis that the extended program would allow for more repetition and participant benefit (Lenze et al., 2014). Taken together, these findings suggest that, because dispositional mindfulness increases as individuals age, condensed programs may be more appropriate for older adults who are able to draw on this strength (Geiger et al., 2016).

To summarize, holistic, cost-effective, proactive, and non-pharmacological approaches, such as MBIs, may have an important role to play in addressing current public health challenges. Indeed, interventions that promote successful aging may allow participants to access a range of benefits including an enhanced sense of calm, resiliency, and perhaps even symptom reduction. Applied proactively, MBIs can improve overall quality of life while at the same time slowing the onset and progression of health-related concerns. Together, these beneficial outcomes can ripple out from the mindful individual to society at large (Lomas et al., 2016).

\section{Barriers to MBI Interventions}

Understanding the concept of mindfulness, let alone how to measure it, is a significant barrier to MBIs that may influence participant attraction, learning, and retention. For instance, the term mindfulness is often considered a new age "buzzword" that is subject to many myths and misinterpretations (Lomas et al., 2016, p. 634). The concept is also subject to the belief that mindfulness is "prohibitively difficult and inaccessible" to the average individual (p. 634).

In the small pilot study using MLOA, barriers to engaging participants in the practice of mindfulness were summarized as procedural (i.e., lack of precise and structured guidelines), numbing (i.e., lack of emotion or sensation during the practice), uncomfortable (i.e., presence of physical pain and annoyances), and "dark" (i.e., presence of difficult and frightening thoughts) (Lomas et al., 2016, p. 635). 
Further, independent of age, meditation, a key component of mindfulness training, is a difficult skill to learn and practice, which poses a significant barrier (Lomas et al., 2015). An interesting study that aimed to highlight the experiential challenges of meditation experienced by adult men makes this point and finds similar barriers to those identified in the MLOA pilot study. Future clinicians and researchers who can better understand these challenges may facilitate a more informed, safe, and supported practice for participants, regardless of age and gender. Further, these findings gave rise to an ethics control recommendation proposing future facilitators allot time after practice sessions to offer participant support in response to any discomfort triggered by meditation practices (Lomas et al., 2016).

Although research in this review focused on older adults, barriers encountered by participants learning to meditate and live more mindfully are universal to MBIs, regardless of participant age. To inform the future delivery of interventions tailored to older adults, addressing these barriers are discussed below.

\section{Tailoring MBIs for Older Adults}

The research in this review offers valuable insights and guidance to scholars, facilitators, and clinicians who plan to deliver MBIs to older adults. In teaching mindfulness, it is important to demystify the concept early on to address common myths such as "mindfulness requires an emptying of the mind" (Lomas et al., 2016, p. 638). When introducing the practice of mindfulness, highlighting its simple integration into everyday life is also recommended at the outset; this strategy can be accomplished easily by providing relevant examples of activities such as appreciating flowers or trees on a walk. Further, early emphasis on how the practice of mindfulness can be slotted into anyone's regular routine, right at home, with a minimal time investment (e.g., 10 minutes) is recommended. The assignment of easy-to-accomplish and realistic homework is also encouraged to allow participants the opportunity to find themselves over-delivering once they sit down to practice.

During mindfulness practice, precise and structured guidance is recommended with, and balanced by, facilitator appreciation of what might be considered intrusive (Lomas et al., 2016). For instance, participants in the MLOA study welcomed the counting of breaths; however, they did not appreciate instruction that surfaced at times during exercises. This feedback suggests that conducting regular pulse-checks with participants could be beneficial; specifically, this feedback would allow facilitators to gain greater insight and consensus to tailor the nature and structure of guidance provided for the remainder of the program.

With a range of limitations faced by older adults, modification to protocols that enhance feasibility should be incorporated into the delivery of MBIs (Geiger et al., 2016). Specifically, modifications that address any physical limitations, health concerns, as well as scheduling and travel constraints should be considered to ensure the comfort and safety of participants. Many studies tailored MBSR and MBCT interventions by simplifying yoga sessions, as well as shortening retreats, sessions, sitting meditations, and homework assignments. One study altered a walking meditation component to avoid the risk of participants losing balance, and provided pillows and blankets to support body exercises, as well as offered options to sit or lie down during certain exercises (Foulk et al., 2014).

The guidance offered in these studies demonstrates the range of possible accommodations and supports to enhance the acceptability of MBIs when delivered to older adults (Foulk et al., 2014). Further research with this older demographic that promotes the standardization of these 
modifications can allow for improved comparability across studies while strengthening empirical support (Geiger et al., 2016).

\section{Research Limitations}

As previously highlighted, many of the traditional MBIs in this review were modified to address the needs of older adult participants; however, in doing so, these studies lack consistency, which limits their research comparability, strength of findings, and capacity to draw meaningful conclusions about the effectiveness of these modifications (Geiger et al., 2016).

In addition to inconsistent modifications, many studies in this review noted a lack of participant diversity. For instance, the MLOA pilot study lacked diversity among its small group of participants, who were privileged, female, educated, reasonably healthy, actively engaged in the community, and highlighted the need for future research to include larger samples of intentionally diverse participants to strengthen findings (Lomas et al., 2016). This lack of participant diversity was also noted in other studies that included predominantly well educated, Caucasian, female participants (Geiger et al., 2016; Mahlo \& Windsor, 2020). Specifically, participants in the 2020 study on dispositional traits were comprised of mostly female (62\%) and Caucasian (80.7\%) participants - most with post-secondary education (75\%) (Mahlo \& Windsor, 2020). Further, as previously noted, inclusion criteria for both Lomas et al.'s (2016) MLOA study as well as Geiger et al.'s (2015) review was based on a chronological age cut-off of 65, which fails to address the diversity of older adults and, beyond, highlights the difficultly in specifically defining this demographic for the purposes of this review.

Also, data reliability concerns were raised corresponding to qualitative data collection using self-report in the MLOA pilot study and the quantitative surveys used in the 2020 study on dispositional traits as well as the 2016 review, all subject to social-desirability biases (Geiger et al., 2016; Lomas et al., 2016; Mahlo \& Windsor, 2020). Further, the study by Mahlo and Windsor (2020) on dispositional traits used a cross-sectional analysis that confounded the effects of age and cohort. Additionally, participants in Mahlo and Windsor's study were recruited via an online research management platform, which raises concerns of sample representativeness due to correlations between advancing age and lower levels of internet use (Mahlo \& Windsor, 2020; Pruchno, 2019).

Another important research limitation found was the inability to determine whether the gains in self-reported participant health and well-being were attributable to group engagement in the studies or the actual practice of mindfulness itself (Geiger et al., 2016). Further, research suggests "mutual, reciprocally enhancing relationships between daily mindfulness and positive emotions" making it difficult to untangle associations between the characteristics of mindfulness and overall well-being (Du et al., 2019 as cited in Mahlo \& Windsor, 2020, p. 9). Lastly, as previously noted, there remains ongoing debate around the definition and optimal measurement of mindfulness, which presents both a barrier and limitation to research on MBIs (Grossman, 2019).

The limitations revealed in this review brought to surface many important considerations for future research in the MBI field. Lack of intervention consistency, participant diversity, reliability risks resulting from self-report measures, and confounding effects can inform the structure and design of future studies that add to this growing body of literature.

\section{Future Research}


With MBI research still in its infancy, the studies selected in this review successfully "plant a seed" and offer guidance to inform future research that targets an older adult demographic (Lomas et al., 2016, p. 639). On one hand, the need to focus research on an older adult population is a high priority; however, from a developmental perspective, interventions that include a broad age-range to evaluate treatment effects across the lifespan is also in need (Mahlo \& Windsor, 2020).

To untangle the associations between the characteristics of mindfulness and overall wellbeing touched on above, future research might consider looking at within-person processes using alternative assessment strategies such as momentary assessment (i.e., sampling experience in realtime) while applying mindfulness to everyday life (Blanke \& Brose, 2017 as cited in Mahlo \& Windsor, 2020).

An additional research path identified in the small MLOA pilot study was the need for future research to focus on linguistic attention in the context of mindfulness training and practice (Lomas et al., 2016). This study revealed participant acceptance of and resistance to certain language used when learning about mindfulness. As an example, participants accepted similes, such as "breath being like a river," but resisted metaphors, such as "take [your] awareness to different parts of [your] body," which they could not comprehend when understood literally (p. 638).

While mindfulness can benefit all people, the limitations identified in this research highlight the pressing need to study more vulnerable and diverse populations (e.g., across ethnicities, genders, and socio-economic statuses) (Hazlett-Stevens et al., 2020; Lomas et al., 2016). The acceptability and feasibility of MBIs demonstrated in this research allow future studies to shift toward well-designed, rather than exploratory, outcomes that continue to advance knowledge in this important field (Berk et al., 2017).

\section{Conclusion}

Given the demands on health care rising in pace with a rapidly aging demographic, wellreceived, holistic, cost-effective, and non-pharmacological approaches to care are necessary, and MBIs propose to meet these extensive criterion (Hazlett-Stevens et al., 2019). Despite the important barriers and limitations identified in this MBI review, which serve to strengthen future research, the possible benefits and opportunities revealed are far too attractive to ignore.

MBIs offer multi-dimensional, evidence-based, and complementary treatment approaches for a vast array of burdens faced by older adults, and, from a successful aging perspective, these interventions have just begun to demonstrate equally vast benefits for mindful individuals, those around them, and society at large (Hazlett-Stevens et al., 2019; Lomas et al., 2016). The strengths of MBIs for older adults may rest in how this demographic relates to looming health-related concerns with enhanced well-being and the ability to make better lifestyle choices (Berk et al., 2017).

As Lomas et al. aptly observes, "[m]indfulness may function like a 'master key', affecting multiple components of well-being" (2016, p. 631). With so many overlapping and beneficial effects, disregarding this approach to well-being is akin to setting this "master key" aside instead of unlocking the possibilities that accompany a more mindful way of life (p. 631). From a public health perspective, there is a non-debatable urgency to address the challenges prompted by an aging demographic; however, from a successful aging outlook, the pursuit of "add[ing] more life 
to years, [and] not just more years to life" is equally vital and MBIs propose to do just that (Vaillant, 2004, p. 561). 


\section{References}

Berk, L., van Boxtel, M., \& van Os, J. (2017). Can mindfulness-based interventions influence cognitive functioning in older adults? A review and considerations for future research. Aging \& Mental Health, 21(11), 1113-1120. https://doi.org/10.1080/13607863.2016.1247423

Brandtstadter, J. (2009). Goal pursuit and goal adjustment: Self-regulation and intentional selfdevelopment in changing developmental contexts. Current Perspectives on Aging and the Life Cycle, 14(1-2), 52-62. http://doi.org//10.1016/j.alcr.2009.03.002

Carstensen, L. L. (2006). The influence of a sense of time on human development. American Association for the Advancement of Science, 312(5782), 1913-1915. http://doi.org//10.1126/science.1127488

Charles, S. T. (2010). Strength and vulnerability integration: A model of emotional well-being across adulthood. Psychological Bulletin, 136(6), 1068-1091. http://doi.org//10.1037/a0021232

Crane, R. S., Brewer, J., Feldman, C., Kabat-Zinn, J., Santorelli, S., Williams, J. M. G., \& Kuyken, W. (2017). What defines mindfulness-based programs? The warp and the weft. Psychological Medicine, 47(6), 990-999. https://doi.org/10.1017/S0033291716003317

de Frias, C. M., \& Whyne, E. (2015). Stress on health-related quality of life in older adults: The protective nature of mindfulness. Aging \& Mental Health, 19(3), 201-206. https://doi.org/10.1080/13607863.2014.924090

Du, J., An, Y., Ding, X., Zhang, Q., \& Xu, W. (2019). State mindfulness and positive emotions in daily life: An upward spiral process. Personality and Individual Differences, 141, 5761. https://doi.org/10.1016/j.paid.2018.11.037

Foulk, M. A., Ingersoll-Dayton, B., Kavanagh, J., Robinson, E., \& Kales, H. C. (2014). Mindfulness-based cognitive therapy with older adults: An exploratory study. Journal of Gerontological Social Work, 57(5), 498-520. https://doi.org/10.1080/01634372.2013.869787

Geiger, P. J., Boggero, I. A., Brake, C. A., Caldera, C. A., Combs, H. L., Peters, J. R., \& Baer, R. A. (2016). Mindfulness-based interventions for older adults: A review of the effects on physical and emotional well-being. Mindfulness, 7(2), 296-307. https://doi.org/10.1007/s12671-015-0444-1

Grossman, P. (2019). On the porosity of subject and object in 'mindfulness' scientific study: Challenges to 'scientific' construction, operationalization and measurement of mindfulness. Current Opinion in Psychology, 28, 102-107.

doi:10.1016/j.copsyc.2018.11.008 
Hazlett-Stevens, H., Singer, J., \& Chong, A. (2019). Mindfulness-based stress reduction and mindfulness-based cognitive therapy with older adults: A qualitative review of randomized controlled outcome research. Clinical Gerontologist, 42(4), 347-358. https://doi.org/10.1080/07317115.2018.1518282

Hempel S., Taylor S. L., \& Marshall, N. J. (2014). Evidence map of mindfulness. Department of Veterans Affairs (US).

Kabat-Zinn, J. (2003). Mindfulness-based interventions in context: Past, present, and future. Clinical Psychology, 10(2), 144-156. http://doi.org//10.1093/clipsy/bpg016

Kocovski, N. L., Segal, Z.V., \& Battista, S.R. (2009). Mindfulness and psychopathology: Problem formation. In Didonna F. (Ed.), Clinical handbook of mindfulness. Springer. https://doi.org/10.1007/978-0-387-09593-6

Lenze, E. J., Hickman, S., Hershey, T., Wendleton, L., Ly, K., Dixon, D., Doré, P., \& Wetherell, J. L. (2014). Mindfulness-based stress reduction for older adults with worry symptoms and co-occurring cognitive dysfunction. International Journal of Geriatric Psychiatry, 29(10), 991-1000. https://doi.org/10.1002/gps.4086

Lomas, T., Cartwright, T., Edginton, T., \& Ridge, D. (2015). A qualitative analysis of experiential challenges associated with meditation practice. Mindfulness, 6(4), 848-860. https://doi.org/10.1007/s12671-014-0329-8

Lomas, T., Ivtzan, I., \& Yong, C. Y. (2016). Mindful living in older age: A pilot study of a brief, community-based, positive aging intervention. Mindfulness, 7(3), 630-641. https://doi.org/10.1007/s12671-016-0498-8

Mahlo, L., \& Windsor, T. D. (2020). Older and more mindful? Age differences in mindfulness components and well-being. Aging \& Mental Health, 25(7),1-12. https://doi.org/10.1080/13607863.2020.1734915

Pruchno, R. (2019). Technology and aging: An evolving partnership. The Gerontologist, 59(1), 1-5. https://doi.org/10.1093/geront/gny153

Rowe, J., \& Kahn, R. (1997). Successful aging. The Gerontologist, 37(4), 433-440. https://doi.org/10.1093/geront/37.4.433

Vaillant, G. (2004). Positive aging. In Linley, P. A., \& Joseph, S. (Eds.), Positive psychology in practice (pp. 561-578). Wiley. http://doi.org//10.1002/9780470939338.ch34

Wingate, L. R., Van Orden, K. A., Joiner, T. E., Williams, F. M., \& Rudd, M. D. (2005). Comparison of compensation and capitalization models when treating suicidality in young adults. Journal of Consulting and Clinical Psychology, 73(4), 756-762. https://doi.org/10.1037/0022-006X.73.4.756 
World Health Organization. (1948, April 7, Reproduced 2021). Constitution. Retrieved March 4, 2021, from https://www.who.int/about/who-we-are/constitution

Xie, J., Zhou, J., Gong, L., Iennaco, J. D., \& Ding, S. (2014). Mindfulness-based cognitive therapy in the intervention of psychiatric disorders: A review. International Journal of Nursing Sciences, 1(2), 232-239. http://doi.org//10.1016/j.ijnss.2014.05.015 